\title{
Quelques questions catholiques à des confrère andrologues
}

\author{
J.M. GueulletTe \\ Religieux dominicain, Théologien moraliste, Docteur en médecine
}

\section{La place du discours religieux sur la sexualité dans une société pluraliste.}

Il semble difficile à certains d'envisager que les représentants des principales religions présentes dans notre pays puisse participer, comme tels, à un débat sur l'éthique et le sens des traitements de l'impuissance masculine. Au débat fréquent sur la laïcité, et sur le confinement éventuel du religieux dans la sphère privée, il me semble que le sujet qui nous rassemble apporte des dimensions particulières.

Le religieux, pas plus que la sexualité, ne peuvent sérieusement être cantonnés dans la sphère privée.

On peut en effet, dans un premier temps, s'étonner que le religieux soit prié de rester à la place que certains lui assignent - c'est à dire la vie privée, la conviction personnelle qui n'a pas à être débattue socialement - quand le sujet de notre débat, la sexualité, fait l'objet d'une large débat social et médiatique. Quoi de plus privé, de plus intime pourtant que la sexualité ? Il me semble intéressant de souligner que dans l'un et l'autre cas, religion et sexualité, il est tout à fait illusoire de croire que l'on puisse limiter la question à une affaire de choix et de comportement privé, comme si la société n'avait aucune part à ce qui est en jeu. La sociologie et l'anthropologie ont largement montré que les divers types de société humaine ont mis en place des formes de régulation sociale de la sexualité, de façon à en limiter les potentialités violentes, et à établir des règles de protection du plus faible et de la communauté. Inversement, la société se trouve requise, mise en cause, et transformée par la sexualité de ses membres, qui lui apporte de nouvelles dimensions, par la procréation, de nouvelles formes de liens sociaux, par l'établissement de liens matrimoniaux entre communautés sociales diverses etc. Les débats actuels sur le contrat d'union civile montrent bien que la sexualité laissée à elle-même au nom de la liberté, dans la sphère purement privée aboutit, tôt ou tard, à des situations d'injustice qui exigent une intervention politique.

La religion connaît exactement le même type d'interactions avec la société : elle nécessite une régulation sociale afin que la communauté humaine au sens le plus large, et donc le plus pluraliste, veille sur la protection de chacun de ses membres, au nom de valeurs qui sont reconnues par tous, et qui dépassent celles de telle ou telle communauté religieuse. Mais la société ne peut se tenir quitte de ce rôle là. Elle est également tributaire à l'égard des religions de ce que celles-ci ont à lui apporter dans le domaine de la réflexion sur le sens de la vie, sur le respect de la personne humaine. La laïcité dans ce qu'elle a de plus anti-religieux est bien souvent une forme d'amnésie à l'égard de tout ce que la tradition judéo-chrétienne a introduit dans la culture occidentale et dans les structures juridiques et politiques de notre société.

Sexe et religion mettent en jeu des réactions et des motivations parfois très proches. 
Un deuxième point mérite, me semble-t-il, d'être souligné. Il s'agit de la prise en compte des relations complexes qu'entretiennent sexualité et religion dans le comportement, l'imaginaire et les croyances des patients que rencontrent les sexologues.

Les mécanismes biologiques et psychologiques qui ont pour finalité la relation à l'autre et la transmission de la vie sont en étroite connexion avec les croyances que chacun a pu développer sur le sens de sa propre vie et sa place dans le monde. Les connexions intimes entre l'impuissance, la stérilité et la mort, sont éclairées d'une manière toute spéciale par la manière dont le patient envisage la mort, l'au-delà, la fécondité de sa propre vie. Les difficultés rencontrées dans la relation sexuelle ne peuvent être prise en compte de manière globale sans une attention portée au sens donné à la sexualité, à la place qui lui est donné dans une appréhension plus large de l'existence, de son sens, des relations amoureuses.

\section{Le discours religieux n'est pas exclusivement normatif}

Ces deux aspects de la place que peut occuper le religieux dans un débat sur la sexualité ne viennent nullement donner à celui-ci une place normative. Bien souvent, certains réagissent à ce niveau, en récusant, à bon droit, à une Église le pouvoir de légiférer pour l'ensemble de la société. Le propos n'est certainement pas ici de l'ordre de la normativité, mais de l'ordre de la participation à une réflexion sur le sens et les modalités d'un certain nombre de thérapeutiques. Restant à leur place dans un débat qui donne la parole à de multiples acteurs sociaux, les religions peuvent apporter leur propre regard sur ce qu'est l'être humain et ce qui peut concourir à son bonheur et à sa dignité.

\section{Pour la communauté catholique, les gestes de la sexualité ont un sens qui les dépasse.}

Sur le sujet qui nous occupe, il faut d'abord rappeler un principe fondamental pour des chrétiens : les actes de la sexualité ont un sens qui dépasse largement la dimension physiologique. Les chrétiens ne sont pas les seuls à avoir remarqué que l'on ne saurait aborder la sexualité de manière juste en en limitant l'étude à des mécanismes hormonaux, vasculaires et biochimiques. Les surprises thérapeutiques, les décisions parfois surprenantes de patients traités pour stérilité, les résistances inexplicables à certaines thérapeutiques sont là pour rappeler au praticien que, là plus encore qu'ailleurs en médecine, l'être humain se révèle dans son unité, dans la complexité des interactions qui structurent son comportement.

\section{L'union sexuelle, expression d'une relation}

A cette intégration, constatable cliniquement, de la sexualité dans des systèmes complexes d'interactions psychiques, la tradition chrétienne ajoute une note particulière qui est celle du sens de ce qui est vécu là. Pour faire bref, on pourrait dire que, pour nous, la sexualité est lorsque elle est pleinement humaine, juste, à sa place - l'expression physique d'un amour qui la dépasse. Si la tradition théologique et la pratique catholique donnent la priorité à la parole échangée, à l'engagement verbalisé, que l'union des corps vient ratifier, d'une certaine manière, il ne faudrait pas en déduire qu'il n'y aurait pas d'interaction en retour. L'expression usuelle " faire l'amour " doit être ainsi comprise dans ses deux sens : le couple fait, c'est à dire réalise, exprime, concrétise, l'amour qui l'unit; mais le couple fait aussi l'amour dans le sens qu'il le construit dans l'acte sexuel lui-même. Ces deux dimensions se retrouvent dans le droit matrimonial catholique, puisque celui-ci précise qu'un mariage qui n'est pas suivi d'une relation sexuelle n'est pas indissoluble, n'est donc pas un mariage chrétien dans lequel les époux se sont tout donné. En revanche, la stérilité n'est pas une cause de nullité de mariage alors que l'impuissance en est une : ce n'est pas la fécondité qui est nécessaire pour nous à l'existence d'un couple véritable mais la possibilité d'une union sexuelle. Celle-ci n'est donc pas uniquement une conséquence de l'amour et de l'engagement mutuel, une expression de celui-ci, elle en est également une condition nécessaire.

L'échec probable de traitements qui ignorent cette approche globale

Une telle approche de la sexualité et de la 
place de celle-ci dans la conception du couple vient éclairer d'une manière assez claire, il me semble, la question du traitement de l'impuissance. Un tel traitement ne peut être envisagé qu'avec intérêt par l'Église catholique s'il peut apporter une aide à des couples en difficultés et les aider à retrouver des conditions satisfaisante d'union sexuelle. Cependant, il n'a des chances d'être pleinement juste d'un point de vue anthropologique, et donc pleinement efficace d'un point de vue clinique, que s'il se situe dans une approche globale de la sexualité et de ses éventuels dysfonctionnements. Je suppose qu'il n'y a pas que les catholiques pour dire qu'un traitement de l'impuissance qui serait envisagé d'un point de vue strictement vasculaire aurait de forte chance d'être souvent inefficace, car il négligerait toutes les dimensions psycho-affectives qui peuvent participer à l'étiologie de l'impuissance. La réticence de la communauté catholique à l'égard de tout ce qui contribue à donner à la sexualité une autonomie, qui la désolidarise du reste de la personne, n'est pas à mettre sur le compte d'un regard méfiant sur la sexualité elle-même, mais sur la conviction que celle-ci perd son sens d'humanité lorsqu'elle n'est plus envisagée dans ses connexions intimes avec tout ce qui fait la personne humaine.

\section{La limite n'est-elle qu'un obstacle à franchir?}

\section{La limite fait partie de l'existence humaine}

Enfin, il faut compléter ce point de vue sur la sexualité par un regard plus large sur ce que sont ces traitements de l'impuissance. L'homme se trouve confronté, dans une telle pathologie, à une limite qui peut remettre profondément en cause son identité et son équilibre. Limpuissance mérite en cela toute l'attention du corps médical et la mise en œuvre de la recherche la plus sérieuse. Mais, ici comme en bien d'autres domaines de la médecine, il faut sans doute se rappeler que l'humanité s'attaque à une limite qu'elle ne pourra de toutes façons repousser indéfiniment. Une attitude juste à l'égard de ces efforts de recherche doit sans doute tenir du paradoxe. Il n'y a aucune raison de ne pas se réjouir des capacités que l'homme développe pour se dépasser, pour repousser les limites que la maladie lui impose. Et l'Église catholique manifeste souvent son soutien à la recherche scientifique et médicale, dont elle se réjouit des avancées. Mais il faut en même temps ne pas perdre de vue que beaucoup de limites qui affectent la vie humaine du fait de la maladie sont, en fait, infranchissables. Linscription de la vie humaine dans l'espace et le temps, l'identité liée à la vie d'un corps humain, sont des données qui ne sont pas dépassables par l'effort scientifique. L'évolution de la médecine au cours des vingt dernières années semble marquée par ce paradoxe : on peut à la fois constater que des victoires considérables ont été remportées, et en même temps remarquer que bien souvent les équipes soignantes se refusent à la mise en œuvre de moyens disproportionnés dans des situations où le malade est proche des limites de la vie. C'est bien souvent dans ces situations limites que se pose de manière cruciale la question du sens de certains gestes thérapeutiques. Peut-être qu'une telle question méritera d'être posée, toutes proportions gardées, devant des demandes de traitement de l'impuissance. Le " droit à l'érection pour tous ", dont il a été question, est-il une revendication qui peut être soutenue sans autre interrogation sur le sens et sur les implications des thérapeutiques qu'il engage?

Y-a-t-il une vie après l'échec thérapeutique?

Un dernier point vient compléter cette interrogation sur la limite. Il serait osé d'affirmer que la tradition chrétienne " ne met pas son salut dans l'orgasme ", selon une expression employée dans notre débat. Au delà d'une petite provocation, cette expression voudrait rappeler qu'il est des limites imposées à nos existences humaines qui ont un caractère plus pathétique que l'impuissance. Mais plus profondément, il s'agit de dire que le rapport chrétien à une limite de ce type ne me semble pas être forcément du côté du franchissement de la limite. Il faut faire tout ce qu'on peut pour traiter le patient, certes, mais en lui gardant la possibilité de trouver sens à sa vie, et à son couple même en cas d'échec. On rencontre la même chose dans le traitement de la stérilité : il faut faire tout ce qu'on peut, et admettre que parfois les couples abandonnent une démarche 
thérapeutique qui leur semble trop lourde, et s'orientent vers une autre manière d'envisager leur avenir et leur fécondité. Dans une société qui revendique haut et fort la victoire qu'elle est censé avoir remporté en se libérant de la normativité religieuse, il convient de se garder du pouvoir tout aussi écrasant que peut s'arroger la norme médicale.

Quelle place laisse-t-on aujourd'hui à celui dont le corps ou le comportement ne sont pas pleinement normaux ou pleinement satisfaisants? N'est-on pas en train d'exiger de l'individu et de la société, au nom de la santé, des sacrifices aussi coûteux que ceux que réclamait autrefois la sainteté ? 